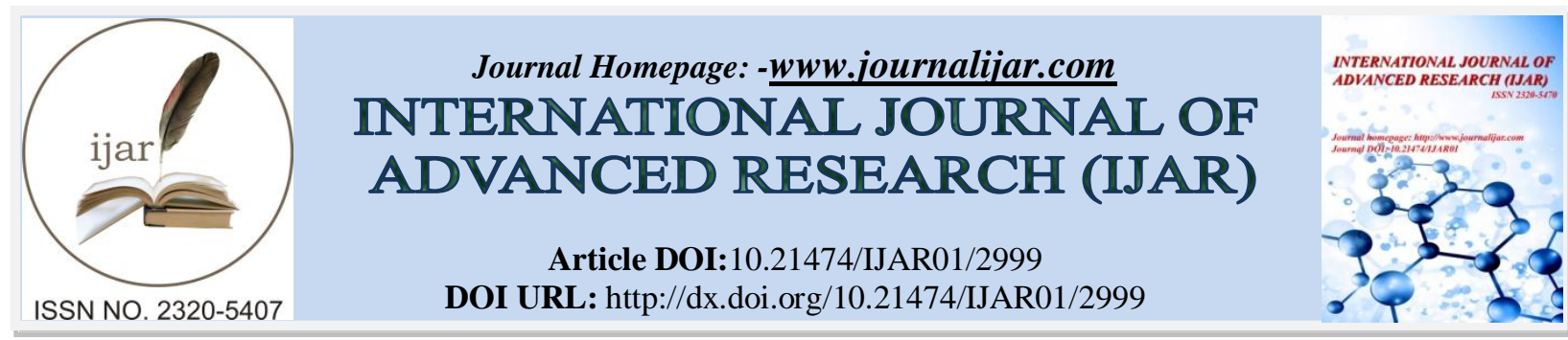

RESEARCH ARTICLE

\title{
ASURVEY AND COMPARISON ON SYNCHRONIZATION METHODS OF CHAOTIC SYSTEMS
}

Mohammad Arabian and Dr. Mohammad Mardani.

Department of Electrical Engineering, ShahabDanesh University, Qom, Iran.

\section{Manuscript Info}

Manuscript History

Received: 28 November 2016

Final Accepted: 27 December 2016

Published: January 2017

Key words:-

Chaos, chaos synchronization, observerbased, Active control, Adaptive Control, Rösslersystem

\begin{abstract}
In this paper, several methods forsynchronization of chaotic systems are explained and compared. The idea is based on drive-response systems synchronization. The methods include: active Control, recursive control, adaptive control, and partial linearizationmethod which are implemented and applied to a Lorenz chaotic system. The partial linearization method is used to synchronize a subset of states of the system to synchronize other states as well. Active control and rebound control methods are used when the system parameters are known while adaptive control method is used when some of the parameters of the system are unknown. Inthesemethods,synchronization is based on Lyapunov stability theory. Three methods, namely, adaptive, active and recursive and are implemented on a $\mathrm{T}$ system successfully. A newmatrix method has been presented for synchronization based on the theory of Lyapanovkrakfskytheory and linear matrix inequality (LMI).This method has been implemented to a Rösslersystem with delay. Comparingto classical methods used to synchronize chaotic system the matrix method seems the best because of easy design of input, suitable for synchronization of chaotic systems with delay, simple calculations, no need to find aLyapunov function for stability.
\end{abstract}

Copy Right, IJAR, 2016,. All rights reserved.

\section{Introduction:-}

Over the years, scientists have tried to invent with different methods, in order to make complex control systems stable and efficient. In this regard, one of the best methods has been proposed by scientists and Russian mathematician, Lyapunov.Lyapunovintroduced a quasi-energy function model for nonlinear systems and then concluded that, if the system energy is constantly reducing, energy finally will finish and the system will be stable.

In this paper, we have investigated the synchronization of chaotic systems. Several methods for synchronization of chaotic systems, namely active control, recursive control, and adaptive control arereviewed and a special matrix method is proposed for designof chaotic system synchronization observer.

The remaining parts of this paper consist of four sections as follows: Inspection 2, an overview of the phenomenon of chaos in the systems is presented with some practical examples of chaotic systems. In section 3, the synchronization of chaotic systems is expressed. Conclusions are expressed in section 4 . 


\section{Chaos phenomenon:-}

Chaos literally means anarchy, confusion and disorder. In chaotic systems, long-term behavior is not possible to predict. This characteristic is the result of high sensitivity of these systems to their initial condition. Chaotic behavior is not random behavior; it is deterministic.[1] But from the view of an observer unaware of the structure and function of chaotic system, the signal cannot be distinguished from a random signal using statistical tests. Since the signal can be measured only with limited precision, even if the chaotic signal generator is completely known, because of uncertainty in initial conditions, the output precision is constantly decreased[1].Therefore, the future of signal, especially in the long-term cannot be predicted and thus, the behavior of signal seems random. Due to sensitivity to initial conditions, a small uncertainty in initial conditions will lead to huge amounts of uncertainty in long term, therefore the system is unpredictable [2].Examples of the chaotic systems include: Burning a neon lamp, the point of incidence and magnitude of earthquakes, the trajectory of the fluid in a tube, population growth organisms, lightning in the sky, and the chain of random numbers generated by computer. $[1,2,3]$. The chaotic systems are studied in several fields of science such as mathematics, astronomy, physics, medicine, meteorology, engineering, mechanics, construction, pharmaceutical and aerospace and even in the fields of psychology, sociology, and management $[2,3]$.

The study of chaos has a lot of applications including:

- Ability to explain many events and natural phenomena, ability to predict disasters and avoid risks of accidents and reduce injuries

- Control of behavior of the systems in the desired direction

- Understanding the process leading to chaos in certain areas and under controlled conditions to achieve optimal conditions[2]

- Developing algorithms and logic of measurement, computing, administrative and operational

- Diagnose the cause of some irregularities, the correction of the laws of science and discovery of new laws[2] Due to the complex and unstable dynamics control, chaos control seems impossible but chaotic systems are capable of self-control and various control objectives are introduced as below:

- Remove chaotic behavior and sustainability point of balance

- $\quad$ stabilization of unstable alternate routes (creation of sustainable Limit Cycle)

- synchronization of two chaotic systems

- iPod control chaos (chaos-chaotic anti-control off)

- Control of bifurcation

\section{Synchronization:-}

Is this paper synchronization of two chaotic systems is examined as follows. It is synchronization of changes in two systems so that they show the same behavior. For example, data transmission in telecommunication systems, both client and transmitter have access to a carrier signal forsynchronization. In 1990, Pecorrat, Carroll showed that in specific circumstances two chaotic systems can become synchronized by applying error signal to systems [1].Assume two identical copies of the dynamical system[2]

$\dot{\mathrm{X}}=\mathrm{f}(\mathrm{x}), \quad \mathrm{x} \in \mathrm{R}^{\mathrm{n}}$

For example, two identical oscillators with different initial conditions. One of these systems is called drive and another one is called response.

Drive System: $\left\{\begin{array}{l}\dot{\mathrm{Z}}_{\mathrm{d}}=\mathrm{Z}\left(\mathrm{Z}_{\mathrm{d}}, \mathrm{y}_{\mathrm{d}}\right) \\ \dot{\mathrm{Y}_{\mathrm{d}}}=\mathrm{Z}\left(\mathrm{Z}_{\mathrm{d}}, \mathrm{y}_{\mathrm{d}}\right)\end{array}\right.$

Response System: $\left\{\begin{array}{l}\dot{Z}_{\mathrm{r}}=\mathrm{Z}\left(\mathrm{Z}_{\mathrm{r}}, \mathrm{y}_{\mathrm{r}}\right) \\ \dot{\mathrm{Y}}_{\mathrm{r}}=\mathrm{Z}\left(\mathrm{Z}_{\mathrm{r}}, \mathrm{y}_{\mathrm{r}}\right)\end{array}\right.$

In equations (2) and (3), $Z_{d}$ and $Y_{d}$ are drive system's state variables andZ $Z_{r} \cdot y_{r}$ are state variables is the answer system.

Synchronization condition is defined as follows:

$\lim _{\mathrm{t} \rightarrow 0}\left|\mathrm{Z}_{\mathrm{d}}(\mathrm{t})-\mathrm{Z}_{\mathrm{r}}(\mathrm{t})\right| \rightarrow 0$

$\lim _{\mathrm{t} \rightarrow 0}\left|\mathrm{y}_{\mathrm{d}}(\mathrm{t})-\mathrm{y}_{\mathrm{r}}(\mathrm{t})\right| \rightarrow 0$ 


\section{Dynamic equations of error signal will be:-}

$\left\{\dot{e_{z}}=f_{0}\left(e_{z}, t\right)\right.$

$\left\{\dot{e}_{\mathrm{y}}=\mathrm{f}_{1}\left(\mathrm{e}_{\mathrm{z}}, \mathrm{e}_{\mathrm{y}}, \mathrm{t}\right)\right.$

Wheree $_{\mathrm{z}}=\mathrm{z}_{\mathrm{d}}-\mathrm{z}_{\mathrm{r}}$ ande $_{\mathrm{y}}=\mathrm{y}_{\mathrm{d}}-\mathrm{y}_{\mathrm{r}}$.

Theorem [4]: Suppose for every initial conditions $z_{d}(0) \operatorname{andz}_{r}(0) \operatorname{andy}_{d}(0)$, responses $z_{d}(t) a n d y=(t)$ in the interval are extremely large and equilibrium point $\mathrm{e}_{\mathrm{z}}=0 \mathrm{ine}_{\mathrm{z}}=\mathrm{f}_{\mathrm{o}}\left(\mathrm{e}_{\mathrm{z}}, \mathrm{t}\right)$ is asymptotically stable in general and uniform.[3] $\dot{e}_{y}=f_{1}\left(e_{z}, e_{y}, t\right)$ is stable. The purpose of synchronization is met then for any initial condition. There are other methods for synchronization, including synchronization with the passive-based, ActiveX control, recursion, adaptive control, partial linearization, and particular matrix methods.[1]

\section{ActiveX control Methods:-}

This method is used to synchronize two systems which are identical (T) in parameters. A system (T) in general is as follows:[5]

T system: $\left\{\begin{array}{c}\dot{x}=a(y-x) \\ \dot{y}=(c-a) x-a x z \\ \dot{z}=-b z+x y\end{array}\right.$

When the coefficients $a=2.1$ and $b=0.6$ and chaotic system is $c=30$ is selected. Synchronization process is as follows. Consider the drive and response systems:

Drive system: $\left\{\begin{array}{c}\dot{\mathrm{x}}_{1}=\mathrm{a}\left(\mathrm{y}_{1}-\mathrm{x}_{1}\right) \\ \dot{\mathrm{y}}_{1}=(\mathrm{c}-\mathrm{a}) \mathrm{x}_{1}-\mathrm{ax}_{1} \mathrm{z}_{1} \\ \dot{\mathrm{z}}_{1}=-\mathrm{bz}_{1}+\mathrm{x}_{1} \mathrm{y}_{1}\end{array}\right.$
Response system: $\left\{\begin{array}{c}\dot{\mathrm{x}}_{2}=\mathrm{a}\left(\mathrm{y}_{2}-\mathrm{x}_{2}\right)+\mathrm{u}_{1}(\mathrm{t}) \\ \dot{\mathrm{y}}_{2}=(\mathrm{c}-\mathrm{a}) \mathrm{x}_{2}-\mathrm{ax}_{2} \mathrm{z}_{2}+\mathrm{u}_{2}(\mathrm{t}) \\ \dot{\mathrm{z}}_{2}=-\mathrm{bz}_{2}+\mathrm{x}_{2} \mathrm{y}_{2}+\mathrm{u}_{3}(\mathrm{t})\end{array}\right.$

The error for the systems is define as:

$\mathrm{e}_{1}=\mathrm{x}_{2}-\mathrm{x}_{1} 9 \mathrm{e}_{2}=\mathrm{y}_{2}-\mathrm{y}_{1}, \mathrm{e}_{3}=\mathrm{z}_{2}-\mathrm{z}_{1}$

\section{The error dynamic equations are obtained as:-}

$\left\{\begin{array}{c}\dot{\mathrm{e}}_{1}=\mathrm{a}\left(\mathrm{e}_{2}-\mathrm{e}_{1}\right)+\mathrm{u}_{1}(\mathrm{t}) \\ \dot{\mathrm{e}}_{2}=(\mathrm{c}-\mathrm{a}) \mathrm{e}_{1}-\mathrm{a}\left(\mathrm{x}_{2} \mathrm{z}_{2}-\mathrm{x}_{1} \mathrm{z}_{1}\right)+\mathrm{u}_{2}(\mathrm{t}) \\ \dot{\mathrm{e}}_{3}=-\mathrm{be}_{3}+\mathrm{x}_{2} \mathrm{y}_{2}-\mathrm{x}_{1} \mathrm{y}_{1}+\mathrm{u}_{3}(\mathrm{t})\end{array}\right.$

$T_{1 h e n u_{1}} a_{d u_{2}} a_{d u}$ are defined control functions for active control like below, error in the dynamic, non-linear segments are eliminated and will be only a function of e.

$\left\{\begin{array}{c}\mathrm{u}_{1}(\mathrm{t})=\mathrm{v}_{1}(\mathrm{t}) \\ \mathrm{u}_{2}(\mathrm{t})=\mathrm{a}\left(\mathrm{x}_{2} \mathrm{z}_{2}-\mathrm{x}_{1} \mathrm{z}_{1}\right)+\mathrm{v}_{2}(\mathrm{t}) \\ \mathrm{u}_{3}(\mathrm{t})=-\mathrm{x}_{2} \mathrm{y}_{2}+\mathrm{x}_{1} \mathrm{y}_{1}+\mathrm{v}_{3}(\mathrm{t})\end{array}\right.$
$\left\{\begin{array}{c}\dot{\mathrm{e}}_{1}=\mathrm{a}\left(\mathrm{e}_{2}-\mathrm{e}_{1}\right)+\mathrm{v}_{1}(\mathrm{t}) \\ \dot{\mathrm{e}}_{2}=(\mathrm{c}-\mathrm{a}) \mathrm{e}_{1}+\mathrm{v}_{2}(\mathrm{t}) \\ \dot{\mathrm{e}}_{3}=-\mathrm{be}_{3}+\mathrm{v}_{3}(\mathrm{t})\end{array} \rightarrow\left[\begin{array}{c}\dot{\mathrm{e}}_{1} \\ \dot{\mathrm{e}}_{2} \\ \dot{\mathrm{e}}_{3}\end{array}\right]=\left[\begin{array}{ccc}-\mathrm{a} & \mathrm{a} & 0 \\ \mathrm{c}-\mathrm{a} & 0 & 0 \\ 0 & 0 & -\mathrm{b}\end{array}\right]\left[\begin{array}{c}\mathrm{e}_{1} \\ \mathrm{e}_{2} \\ \mathrm{e}_{3}\end{array}\right]+\left[\begin{array}{c}\mathrm{v}_{1} \\ \mathrm{v}_{2} \\ \mathrm{v}_{3}\end{array}\right]\right.$

Now, it is sufficient to calculate the control vector $\mathbf{v}$, depending on the state variables so that the error is diminished.

Thatise $=-$ ethe response of the equation is $\mathrm{e}^{-\mathrm{t}}$. That is theerrors approach zero when $\mathrm{t} \rightarrow \infty[16]$

The matrix $\mathrm{A}$ is chosen so that the eigenvalues of matrix $\mathrm{A}$ are -1 .

$\left[\begin{array}{l}\mathrm{v}_{1} \\ \mathrm{v}_{2} \\ \mathrm{v}_{3}\end{array}\right]=\mathrm{A}\left[\begin{array}{l}\mathrm{e}_{1} \\ \mathrm{e}_{2} \\ \mathrm{e}_{3}\end{array}\right] \rightarrow \mathrm{A}=\left[\begin{array}{ccc}\mathrm{a}-1 & -\mathrm{a} & 0 \\ \mathrm{a}-\mathrm{c} & -1 & 0 \\ 0 & 0 & \mathrm{~b}-1\end{array}\right]$

$\left\{\begin{array}{lr}\dot{\mathrm{e}}_{1}=-\mathrm{e}_{1} & \mathrm{e}_{1}(\mathrm{t})=\mathrm{c}_{1} \mathrm{e}^{-\mathrm{t}} \\ \dot{\mathrm{e}}_{2}=-\mathrm{e}_{2} \rightarrow \mathrm{e}_{2}(\mathrm{t})=\mathrm{c}_{2} \mathrm{e}^{-\mathrm{t}} \\ \dot{\mathrm{e}}_{3}=-\mathrm{e}_{3} \mathrm{e}_{3}(\mathrm{t})=\mathrm{c}_{3} \mathrm{e}^{-\mathrm{t}}\end{array}\right.$ 
The initial values for the simulation are:-

$\mathrm{x}_{1}(0)=0.1 ، \mathrm{x}_{2}(0)=2.4 \cdot \mathrm{y}_{1}(0)=-0.3 ، \mathrm{y}_{2}(0)=-3.3 ، \mathrm{z}_{1}(0)=0.2, \mathrm{z}_{2}(0)=14.5$

Figure (1) shows the changes of $x_{1}$ and $x_{2}$ where Figures (2) and (3) showy $y_{1}$ and $y_{2}$ and $z_{1}$ and $z_{2}$ respectively. Figure (4) presentse ${ }_{1}, \mathrm{e}_{2}$ ande $_{3}$ versus time [7].

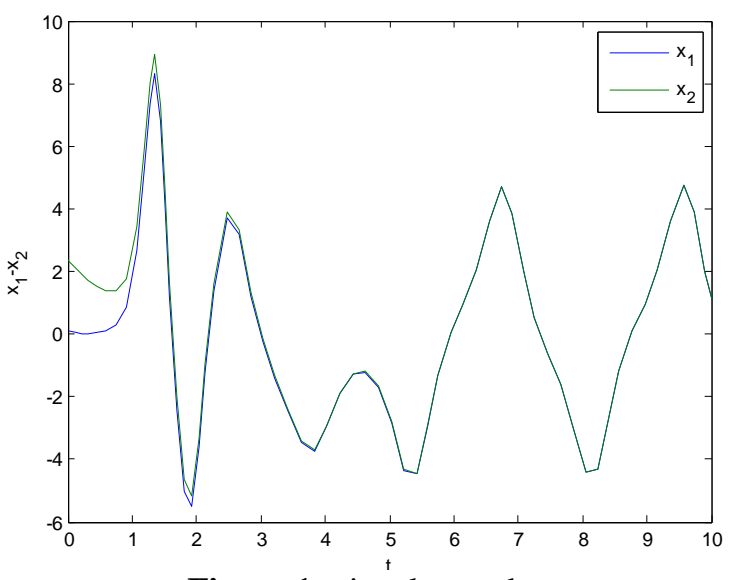

Figure 1:-signals $x_{2}$ and $x_{1}$.

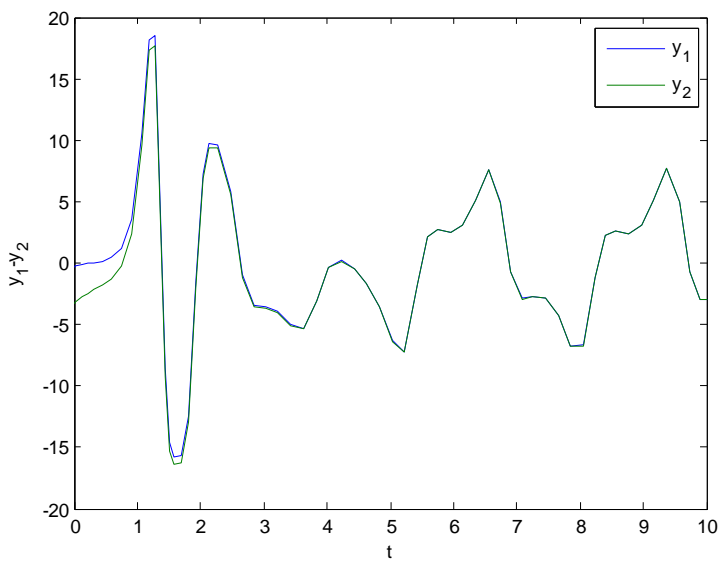

Figure 2:-.Signals $y_{1}$ and $y_{2}$

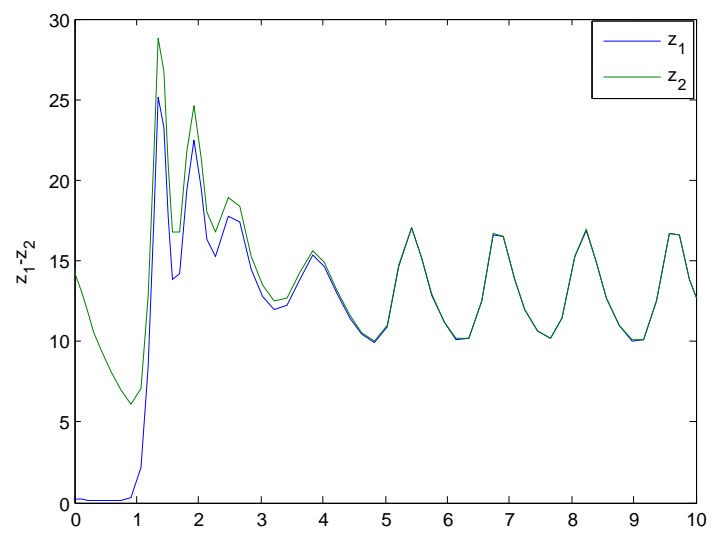

Figure 3:- Signals $z_{1}$ and $z_{2}$ 


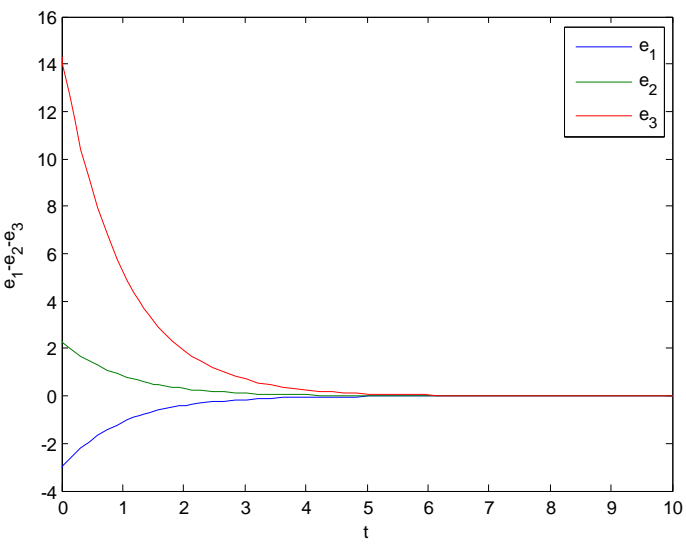

Synchronization by adaptive control

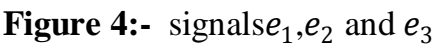

In particular situations, some parameters of the system are unknown. [9]

Once again there where the dynamic equations error as follows: [10]

$\left\{\begin{array}{c}\dot{e}_{1}=a\left(e_{2}-e_{1}\right)+u_{1}(t) \\ \dot{e}_{2}=(c-a) e_{1}-a\left(x_{2} z_{2}-x_{1} z_{1}\right)+u_{2}(t) \\ \dot{e}_{3}=-b e_{3}+x_{2} y_{2}-x_{1} y_{1}+u_{3}(t)\end{array}\right.$

A positive definite Lyapunov function is defined as:-

$v\left(e_{1}, e_{2}, e_{3}, \tilde{a}, \tilde{b}, \tilde{c}\right)=\left(\frac{1}{2}\right)\left(e_{1}^{2}+e_{2}^{2}+e_{3}^{2}+\tilde{a}^{2}+\tilde{b}^{2}+\tilde{c}^{2}\right)$

Where $\tilde{a}=a-a_{1} \cdot \tilde{b}=b-b_{1}$ ‘ $\tilde{c}=c-c_{1} a n d a_{1}, b_{1}, c_{1}$ are the estimated values of the unknown parameters a, $\mathrm{b}, \mathrm{c}$. we assume control functions as:

$\left\{\begin{array}{c}u_{1}(t)=-a_{1}\left(e_{2}-e_{1}\right) \\ u_{2}(t)=-\left(c_{1}-a_{1}\right) e_{1}+a_{1}\left(x_{2} z_{2}-x_{1} z_{1}\right) \\ u_{3}(t)=\left(b_{1}-1\right) e_{3}-x_{2} y_{2}-x_{1} y_{1}\end{array}\right.$

The updating rules Are:-

$\left\{\begin{array}{r}\dot{a}_{1}=-e_{1}^{2}-e_{2}\left(x_{2} z_{2}-x_{1} z_{1}\right) \\ \dot{b}_{1}=-e_{3}^{2} \\ \dot{c}_{1}=e_{1} e_{2}\end{array}\right.$

Then, the derivative of the Function v:-

$v\left(e_{1}, e_{2}, e_{3}, \tilde{a}, \tilde{b}, \tilde{c}\right)=e_{1}^{2}-e_{1}^{2}-e_{1}^{2}<0$

The signals $x_{2}$ and $y_{2}$ and $z_{2}$ are from response system, while $x_{1}$ and $y_{1}$ and $z_{1}$ are synchronized drive system variables.[10]

The initial values for the simulation are as follows:-

$x_{1}(0)=0.1 \cdot x_{2}(0)=2.4 \cdot y_{1}(0)=-0.3 \quad y_{2}(0)=-3.3 \quad z_{1}(0)=0.2 \quad \cdot z_{2}(0)=14.5 \quad, a_{1}(0)=b_{1}(0)=$ $c_{1}(0)=0.1$

Figure (5) shows $x_{1}-x_{2}$, Figure (6) shows $y_{1}-y_{2}$, Figure (7) shows $z_{1}-z_{2}$, and Figure (8) shows $e_{1}$ and $e_{2}$ and $e_{3}$ versus time. 


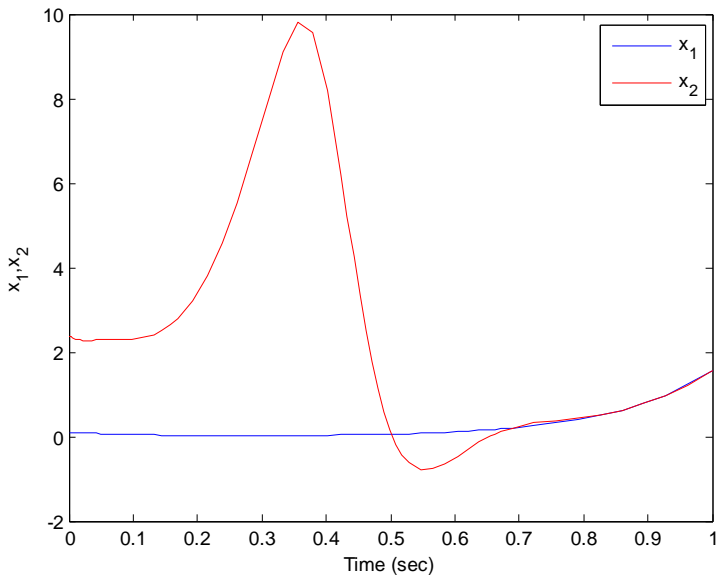

Figure 5:- for $x_{1}-x_{2}$

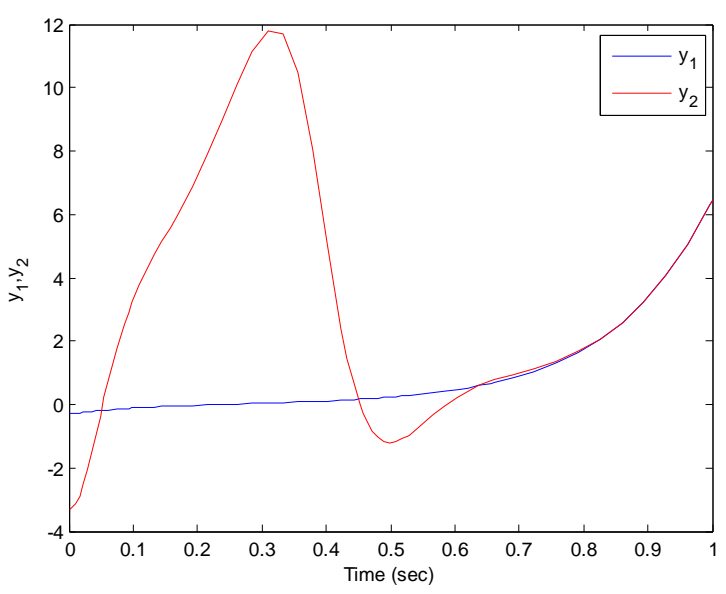

Figure 6:- $y_{1}-y_{2}$

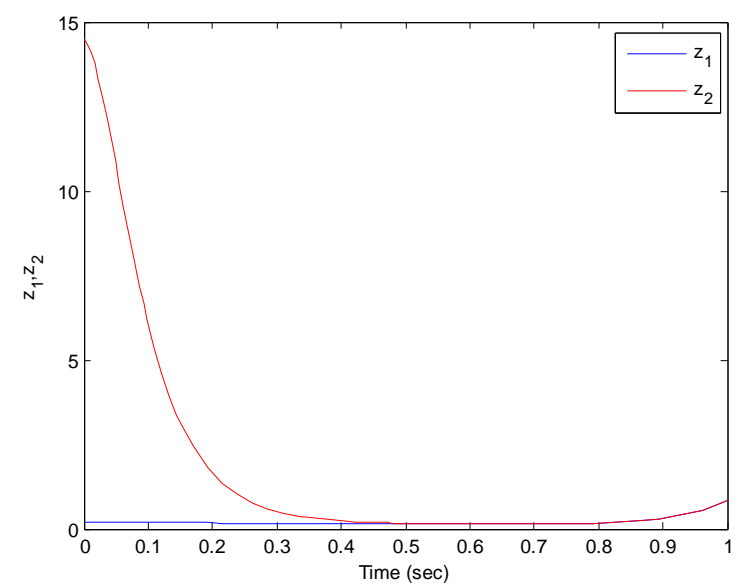

Figure 7:- $z_{1}-z_{2}$ 


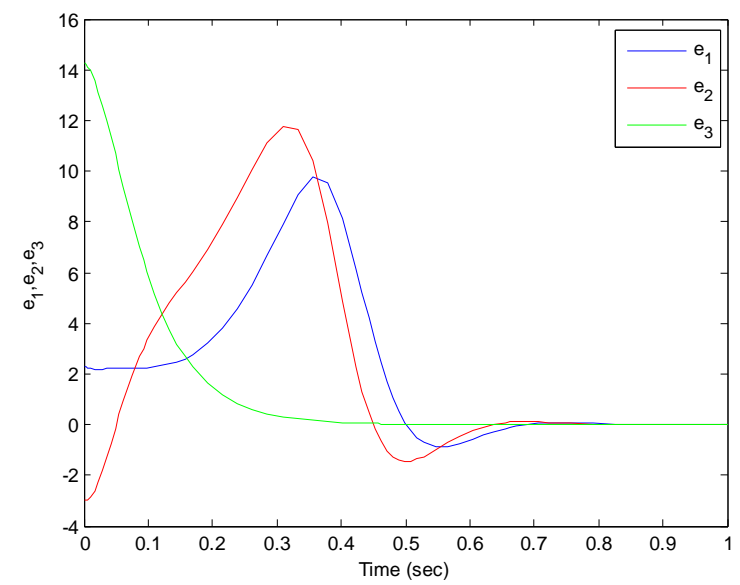

Figure 8:- $. e_{1}, e_{2}$ and $e_{3}$

\section{Synchronization by backstopping:-}

This method is efficient for up to two parameters in the design of the system (T) [11]. The dynamical equation of system error is:

$$
\left\{\begin{array}{c}
\dot{e}_{1}=a\left(e_{2}-e_{1}\right)+u_{1}(t) \\
\dot{e}_{2}=(c-a) e_{1}-a\left(e_{1} e_{3}+x_{1} e_{3}+e_{1} z_{1}\right)+u_{2}(t) \\
\dot{e}_{3}=-b e_{3}+e_{1} e_{2}+e_{1} y_{1}+e_{2} x_{1}+u_{3}(t)
\end{array}\right.
$$

There are three stages in backstopping, in the first step we define $z_{1}=e_{1}$, then:

$\dot{z}_{1}=a e_{2}-a z_{1}+u_{1}(t)$

Considering $\quad e_{2}=\alpha_{1}\left(z_{1}\right)$

as

a

virtual

where $\alpha_{1}\left(z_{1}\right)$ is designed to stabilize the $z_{1}$ in (22), Then $v_{1}$ is chosen as aLyapunov function:

$v_{1}=\frac{z_{1}^{2}}{2}$

$\dot{v}_{1}=z_{1} \dot{z}_{1}=-a z_{1}^{2}+a z_{1} \alpha_{1}+u_{1}(t) z_{1}$

If $u_{1}(t)=0$ and $\alpha_{1}=z_{1}-\frac{z_{1}}{a}$ then $\dot{v}_{1}$ will be negative.

$\alpha_{1}\left(z_{1}\right)$ is a virtual control function. $z_{2}$ is defined as:

$z_{2}=e_{2}-\alpha_{1}$

$\left\{\begin{array}{c}\dot{z}_{1}=a z_{2}-z_{1} \\ \dot{z}_{2}=(c-a) z_{1}-a\left(z_{1} e_{3}+x_{1} e_{3}+z_{1}^{2}\right)-\left(1-\frac{1}{a}\right)\left(a z_{2}-z_{1}\right)+u_{2}(t)\end{array}\right.$

In the next step, thesecondLyapunov function is defined:

$v_{2}=v_{1}+\frac{z_{2}^{2}}{2}$

The derivation to of $v_{2}$ will be:

$\dot{v}_{2}=-z_{1}^{2}-z_{2}^{2}<0$

The functions are chosen $\operatorname{as} \alpha_{2}\left(z_{1}, z_{2}\right)=0$ and $u_{2}=-z_{2}-c z_{1}+a z_{1}^{2}+\left(1-\frac{1}{a}\right)\left(a z_{2}-z_{1}\right)$ then:

$\left\{\begin{array}{c}\dot{z}_{1}=a z_{2}-z_{1} \\ \dot{z}_{2}=-a z_{1}-z_{2}-a\left(z_{1}+x_{1}\right) z_{3} \\ \dot{z}_{3}=-b z_{3}+z_{1} y_{1}+\left(z_{1}+x_{1}\right)\left(z_{2}+z_{1}-\frac{z_{1}}{a}\right)+u_{3}(t)\end{array}\right.$

To thirdLyapunovfunction is $v_{3}$ :

$v_{3}=v_{2}+\frac{z_{3}^{2}}{2}$

The derivative of $v_{3}$ will be: 
$\dot{v}_{3}=-z_{1}^{2}-z_{2}^{2}-a\left(z_{1}+x_{1}\right) z_{2} z_{3}+z_{3} m$

Where

$m=\left[-b z_{3}+z_{1} y_{1}+\left(z_{1}+x_{1}\right)\left(z_{2}+z_{1}+\frac{z_{1}}{a}\right)+u_{3}(t)\right]$

If $u_{3}(t)=(b-a) z_{3}-z_{1} y_{1}+\left(z_{1}+x_{1}\right)\left((a+1) z_{2}+z_{1}-\frac{z_{1}}{a}\right)$ and $\alpha_{2}=0$, then $\dot{v}_{3}<0$ hence the equilibrium point $(0,0,0)$ is asymptotically stable. By taking $z_{1}=e$ ' $z_{2}=e_{2}-\alpha_{1}, z_{3}=e_{3}-\alpha_{2}\left(z_{1}, z_{2}\right)$ it is observed that $e_{1}$ ، $e_{2}$ and $e_{3}$ asymptotically approach zero, that means the synchronization between the systems. The initial values for the simulation are as follows:

$x_{1}(0)=0.1 ، x_{2}(0)=2.4 ، y_{1}(0)=-0.3 ، y_{2}(0)=-3.3 ، z_{1}(0)=0.2 ، z_{2}(0)=14.5$

Figures (9) to (12) show the variables $x_{1}-x_{2}, y_{1}-y_{2}, z_{1}-z_{2}$ and errors $e_{1}, e_{2}$ and $e_{3}$ respectively $[6,7,11]$.

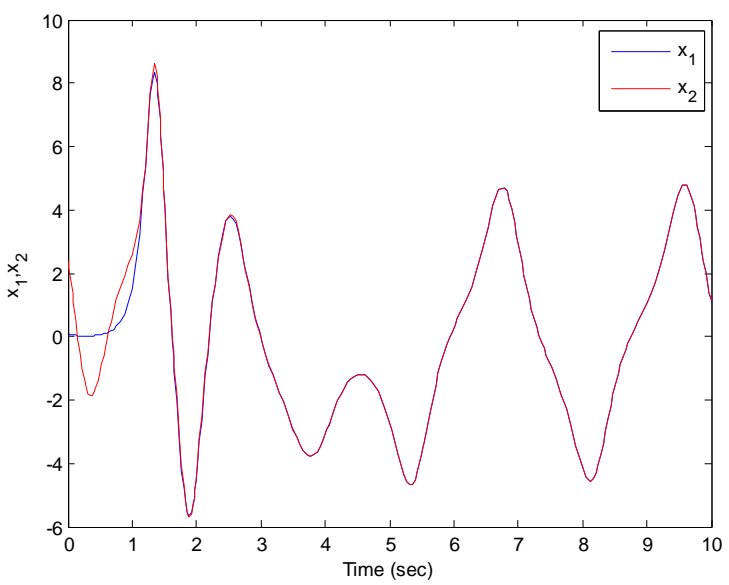

Figure 9:- $x_{1}-x_{2}$

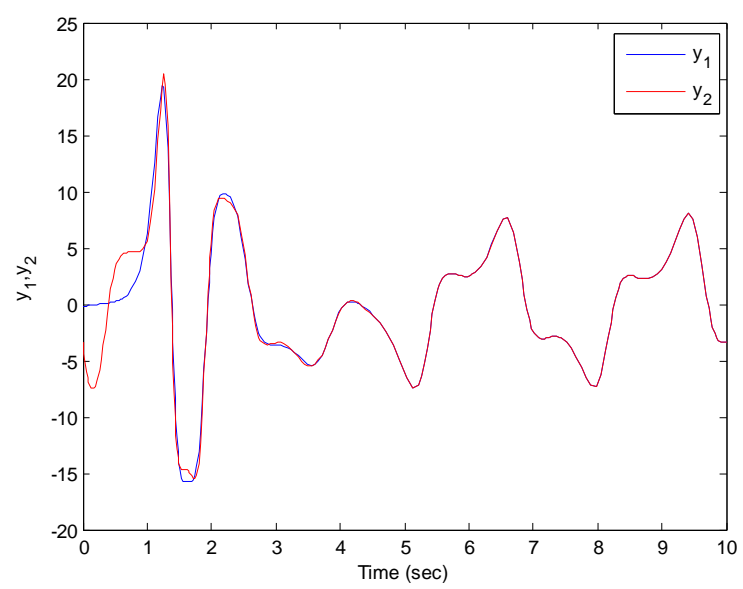

Figure 10:- $y_{1}-y_{2}$ 


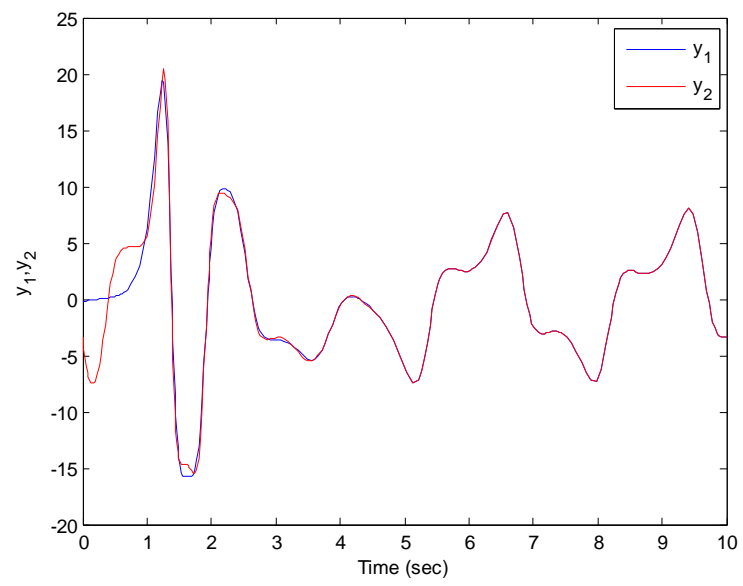

Figure 11:- $z_{1}-z_{2}$

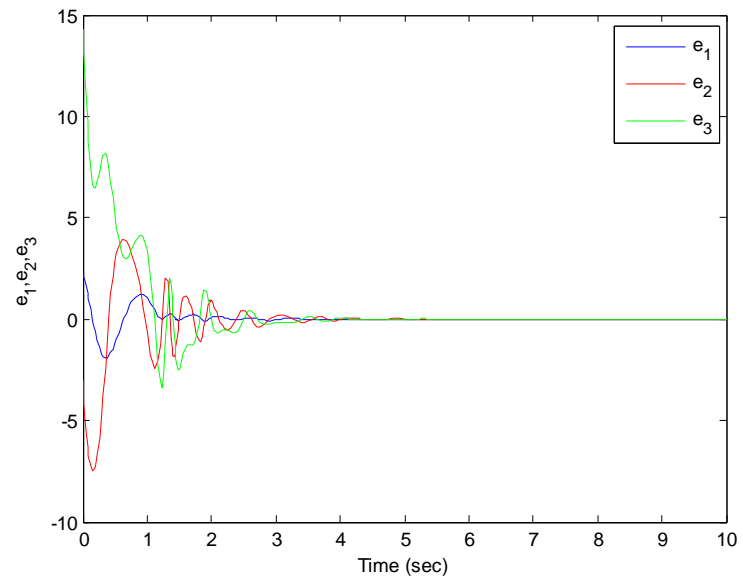

Figure 12:- errors $e_{1}$ and $e_{2}$ and $e_{3}$

Synchronization using the particular matrix:-

This method assumes unknown delay [14]. The equations are as follows for drive and response systems:

$$
\begin{gathered}
\left\{\begin{array}{c}
\dot{x}_{1}(t)=-x_{1}(t)-x_{2}(t)+a_{1}\left[x_{1}\left(t-\tau_{1}\right)\right]+a_{2}\left[x_{2}\left(t-\tau_{2}\right)\right] \\
\dot{x}_{2}(t)=x_{1}(t)+\beta_{1} x_{2}(t)
\end{array}\right. \\
\dot{x}_{3}(t)=\left(x_{1}(t)-\gamma\right) x_{3}(t)+x_{1}(t) x_{3}(t)+\beta_{2} \\
\left\{\begin{array}{c}
\dot{y}_{1}(t)=-y_{1}(t)-y_{2}(t)+a_{1}\left[y_{1}\left(t-\tau_{1}\right)\right]+a_{2}\left[y_{2}\left(t-\tau_{2}\right)\right]-u_{1}(t) \\
\dot{y}_{2}(t)=y_{1}(t)+\beta_{1} y_{2}(t)-u_{2}(t)
\end{array}\right. \\
\dot{y}_{3}(t)=\left(y_{1}(t)-\gamma\right) y_{3}(t)+y_{1}(t) x_{3}(t)-\beta_{2} u_{3}(t)
\end{gathered}
$$

The equations in the form of a matrix can be written as follows:-

$$
\begin{aligned}
& {\left[\begin{array}{l}
\dot{x}_{1}(t) \\
\dot{x}_{2}(t) \\
\dot{x}_{3}(t)
\end{array}\right]=\left[\begin{array}{ccc}
0 & -1 & -1 \\
1 & \beta_{1} & 0 \\
x_{3} & 0 & x_{1}(t)-\gamma
\end{array}\right]\left[\begin{array}{l}
x_{1}(t) \\
x_{2}(t) \\
x_{3}(t)
\end{array}\right]+\left[\begin{array}{ccc}
a_{1} & a_{2} & 0 \\
0 & 0 & 0 \\
0 & 0 & 0
\end{array}\right]\left[\begin{array}{l}
x_{1}\left(t-\tau_{1}\right) \\
x_{2}\left(t-\tau_{2}\right) \\
x_{3}\left(t-\tau_{2}\right)
\end{array}\right]+\left[\begin{array}{l}
0 \\
0 \\
\beta_{2}
\end{array}\right]} \\
& {\left[\begin{array}{l}
\dot{y}_{1}(t) \\
\dot{y}_{2}(t) \\
\dot{y}_{3}(t)
\end{array}\right]=\left[\begin{array}{ccc}
0 & -1 & -1 \\
1 & \beta_{1} & 0 \\
y_{3}(t) & 0 & y_{1}(t)-\gamma
\end{array}\right]\left[\begin{array}{l}
y_{1}(t) \\
y_{2}(t) \\
y_{3}(t)
\end{array}\right]+\left[\begin{array}{ccc}
a_{1} & a_{2} & 0 \\
0 & 0 & 0 \\
0 & 0 & 0
\end{array}\right]\left[\begin{array}{l}
y_{1}\left(t-\tau_{1}\right) \\
y_{2}\left(t-\tau_{2}\right) \\
y_{3}\left(t-\tau_{2}\right)
\end{array}\right]+\left[\begin{array}{l}
0 \\
0 \\
\beta_{2}
\end{array}\right]-\left[\begin{array}{l}
u_{1}(t) \\
u_{2}(t) \\
u_{3}(t)
\end{array}\right]}
\end{aligned}
$$

The values $\tau_{1}$ ' $\tau_{2}$ and $\tau_{3}$ are delay parameters of the system. 
According to data provided by the design of the control input Theorem 1 in [13], obtained control values as follows:-

$u(t)=\left[\begin{array}{ccc}\zeta_{1} & 0 & -1+x_{3}(t) \\ 0 & \zeta_{2} & 0 \\ 0 & 0 & \zeta_{3}\end{array}\right]\left[\begin{array}{l}e_{1}(t) \\ e_{2}(t) \\ e_{3}(t)\end{array}\right]$

The system error equation is obtained as follows:-

$\left[\begin{array}{l}\dot{e}_{1}(t) \\ \dot{e}_{2}(t) \\ \dot{e}_{3}(t)\end{array}\right]=\left[\begin{array}{ccc}\zeta_{1} & -1 & -x_{3}(t) \\ 1 & \beta_{1}-\zeta_{2} & 0 \\ x_{3}(t) & 0 & y_{1}(t)-\zeta_{3}-\gamma\end{array}\right]\left[\begin{array}{l}e_{1}(t) \\ e_{2}(t) \\ e_{3}(t)\end{array}\right]+\left[\begin{array}{ccc}a_{1} & a_{2} & 0 \\ 0 & 0 & 0 \\ 0 & 0 & 0\end{array}\right]\left[\begin{array}{l}e_{1}\left(t-\tau_{1}\right) \\ e_{2}\left(t-\tau_{2}\right) \\ e_{3}\left(t-\tau_{2}\right)\end{array}\right]$

According to Theorem 1 in [14] system error dynamic equations for asymptotic stability will be making the following conditions.

$\left\{\begin{array}{c}-\zeta_{1}+2\left|a_{1}\right|+\left|a_{2}\right|<0 \\ \beta_{1}-\zeta_{2}+\left|a_{2}\right|<0 \\ y_{1}(t)-\gamma-\zeta_{3}<0\end{array}\right.$

The initial values for the simulation are as follows:-

$a_{1}=0.2 \cdot a_{2}=0.5 \cdot \beta_{1}=0.2 \cdot \beta_{2}=0.2 \cdot \gamma=5.7 \cdot \tau_{1}=0.2 \cdot \tau_{2}=0.1 \cdot \zeta_{1}=1 \cdot \zeta_{2}=1 \cdot \zeta_{3}=y_{1}(t)$ $،\left[\begin{array}{lll}x_{1}(0) & x_{2}(0) & x_{3}(0)\end{array}\right]=\left[\begin{array}{lll}-2 & -4 & 10\end{array}\right] \cdot\left[\begin{array}{lll}y_{1}(0) & y_{2}(0) & y_{3}(0)\end{array}\right]=\left[\begin{array}{lll}1 & 2 & 1\end{array}\right]$

Figures (13) to (16) show $x_{1}-y_{1}, x_{2}-y_{2}, x_{3^{-}} y_{3}$ and errors $e_{1}, e_{2}$ and $e_{3}$ respectively [15].

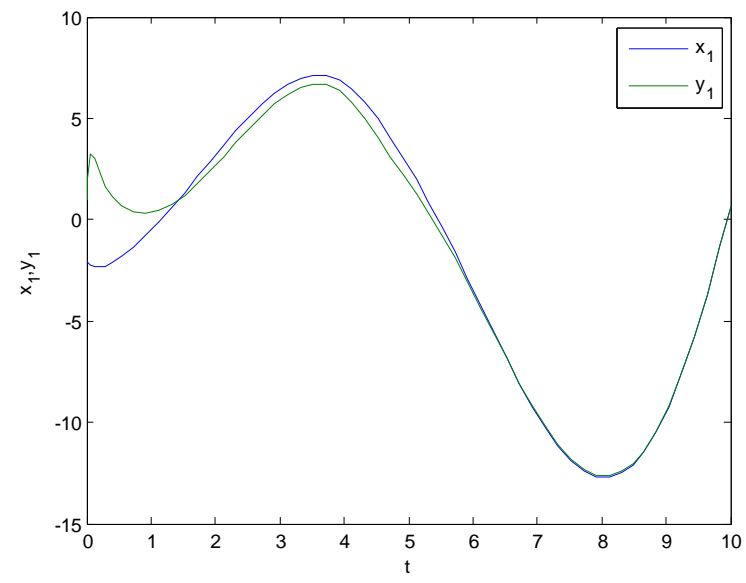

Figure 13:- $x_{1}-y_{1}$

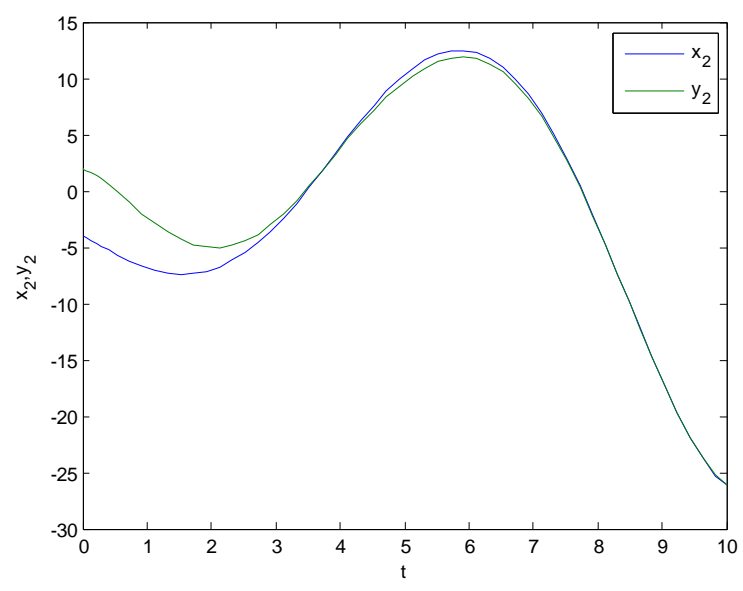

Figure 14:- $x_{2}-y_{2}$ 


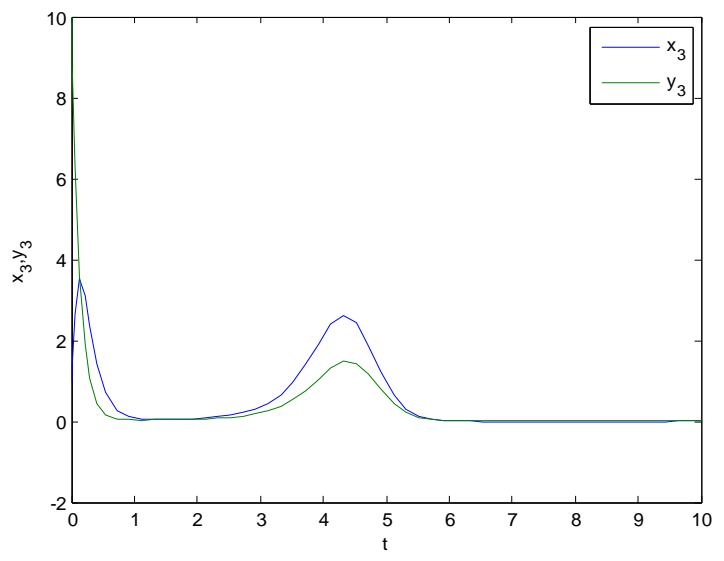

Figure 15:- $x_{3}-y_{3}$

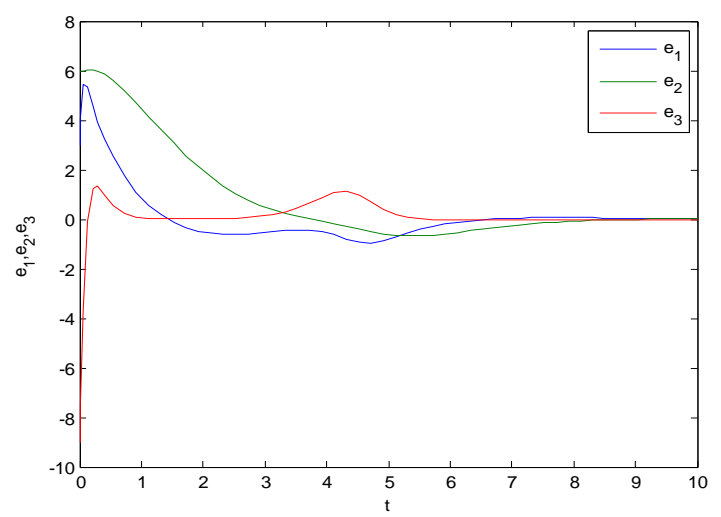

Figure 16:- errors $e_{1}$ and $e_{2}$ and $e_{3}$

\section{linear method Details:-}

In this method, the synchronization of one stateconcludes synchronization of all other states. Integrated system is considered with the following equations:

$$
\left\{\begin{array}{c}
\dot{x}_{1}=(25 \theta+10)\left(y_{1}-x_{1}\right) \\
\dot{y}_{1}=(28-35 \theta) x_{1}-x_{1} z_{1}+(29 \theta-1) y_{1} \\
\dot{z}_{1}=x_{1} y_{1}-\frac{8+\theta}{3} z_{1}
\end{array}\right.
$$

The system will be up for $\theta=0, \theta=0.8$ and $\theta=1$, respectively Lorenz chaotic system, Lu and Chen. Considering the equation (40) as the actuator and the equation (41) as the system's response:

$$
\left\{\begin{array}{c}
\dot{x}_{2}=(25 \theta+10)\left(y_{2}-x_{2}\right) \\
\dot{y}_{2}=(28-35 \theta) x_{2}-x_{2} z_{2}+(29 \theta-1) y_{2}+u \\
\dot{z}_{2}=x_{2} y_{2}-\frac{8+\theta}{3} z_{2}
\end{array}\right.
$$

The error for the system is defined:

$$
e_{1}=x_{2}-x_{1} g e_{2}=y_{2}-y_{1} g e_{3}=z_{2}-z_{1}
$$

Error dynamic equations will be obtained as follows:

$$
\left\{\begin{array}{c}
\dot{e}_{1}=(25 \theta+10)\left(e_{2}-e_{1}\right) \\
\dot{e}_{2}=(28-35 \theta) e_{1}+(29 \theta-1) e_{2}-e_{1} e_{3}-z_{1} e_{1}-x_{1} e_{3}+u \\
\dot{e}_{3}=e_{1} e_{2}+x_{1} e_{2}+y_{1} e_{1}-\frac{8+\theta}{3} e_{3}
\end{array}\right.
$$


So that the control vector $u$ we is chosen as a linear Relationship:-

$u=(35 \theta-28) e_{1}-29 \theta e_{2}+e_{1} e_{3}+z_{1} e_{1}+x_{1} e_{3}$

$\dot{e}_{2}=e_{2}$

According to Theorem 1 in [13]. Implementation wishes to simulate this technique to the Lorenz system as follows:-

$$
\begin{aligned}
& \left\{\begin{array}{c}
\dot{e}_{1}=10\left(e_{2}-e_{1}\right) \\
\dot{e}_{2}=28 e_{1}-1 e_{2}-e_{1} e_{3}-z_{1} e_{1}-x_{1} e_{3}+u \\
\dot{e}_{3}=e_{1} e_{2}+x_{1} e_{2}+y_{1} e_{1}-\frac{8}{3} e_{3}
\end{array}\right. \\
& u=-28 e_{1}+e_{1} e_{3}+z_{1} e_{1}+x_{1} e_{3}
\end{aligned}
$$

The initial values for the simulation are as Follows:-

$$
\begin{aligned}
& {\left[\begin{array}{lll}
x_{1}(0) & y_{1}(0) & z_{1}(0)
\end{array}\right]=\left[\begin{array}{ccc}
10 & 10 & 10
\end{array}\right]} \\
& {\left[\begin{array}{lll}
e_{1}(0) & e_{2}(0) & e_{3}(0)
\end{array}\right]=\left[\begin{array}{lll}
-5 & -10 & 10
\end{array}\right]}
\end{aligned}
$$

Figure (17) to (20) show $x_{1}-x_{2}, y_{1}-y_{2}, z_{1}-z_{2}$ and errors $\mathrm{e}_{1}, \mathrm{e}_{2}$ and $\mathrm{e}_{3}$ respectively[15].

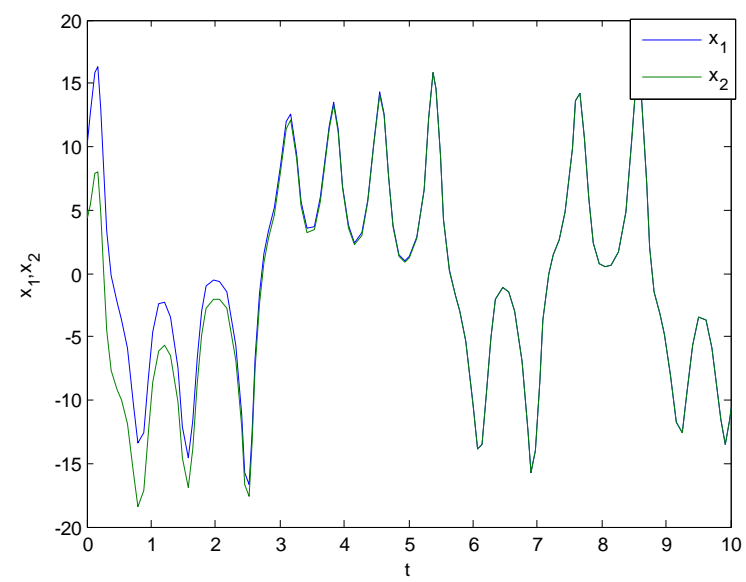

Figure 17:- for $x_{1}-x_{2}$

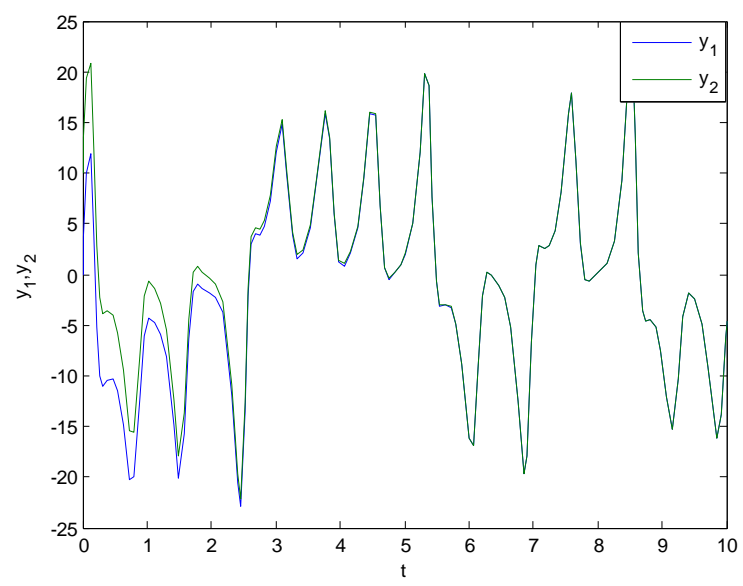

Figure 18:- $y_{1}-y_{2}$ 


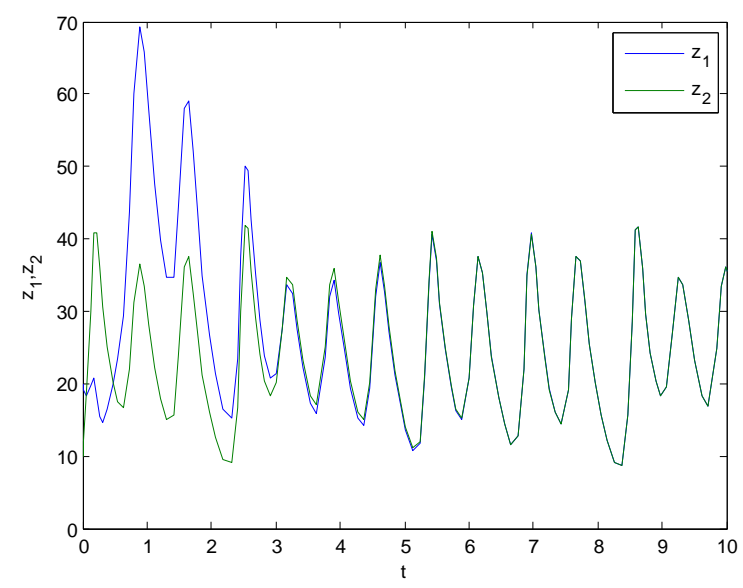

Figure 19:- $\mathrm{z}_{1}-\mathrm{z}_{2}$

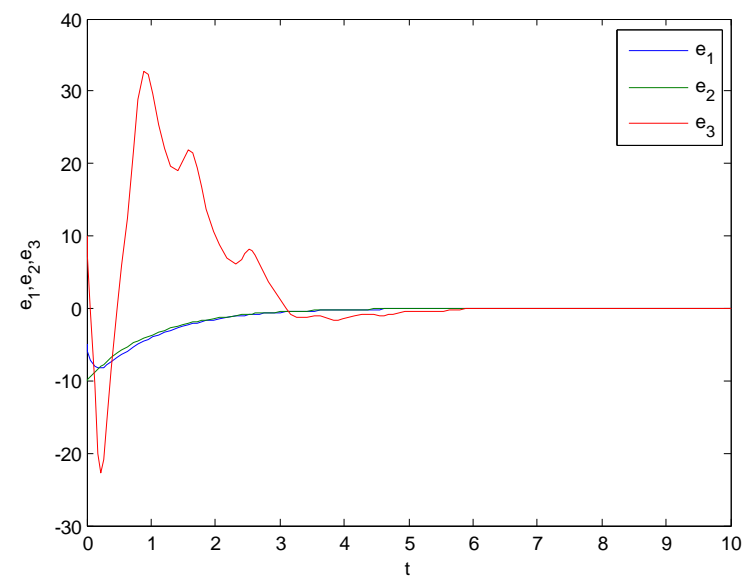

\section{Conclusion:-}

Figure 20:- errors $\mathrm{e}_{1}$ ande $\mathrm{e}_{2}$ and $\mathrm{e}_{3}$

In this paper we dealt with the history of chaos, chaos theory and synchronization of chaotic systems. Synchronization methods used in this paper areActive control, backstopping control, adaptive control, partial linearization matrix method, which is based on synchronization systems with a delay. All these methods are based on Lyapunov theory.

SIMULINK environment of MATLAB software has been utilized for simulation of synchronization of chaos systems. The methods designed for easy synchronization of chaotic systems introduce suitable methods for synchronization of delayed chaotic systems, with simple calculations, and no need to calculations of Lyapunov function for stability in contrast to the classical methods.

\section{References:-}

1. S Wiggins,[BOOK] Introduction to applied nonlinear dynamical systems and chaos, 2003

2. WilfridPerruquetti ,Jean-Pierre barbot [Book] Chaos in Automatic Control,2005

3. HerveDedieu,peterkennedy,martinhasler, Chaos shift Keying; Modulation and Demodulation of a Chaotic Carrier Using Self-synchronizing Chua's Circuits, IEEE Transactions onCircuits and Systems (1993) 634-643.

4. T.L. Carroll, L.M. Pecora, Synchronizing chaotic circuits, IEEE Transactions onCircuits and Systems 38 (4) (1991) 453-456.

5. G. Grassi, S. Mascolo, Nonlinear observer design to synchronize hyperchaotic systems via a scalar signal, IEEE Transactions on Circuits and Systems Fundamental Theory and Applications 44 (10) (1997) 1011-1014.

6. Alligood Kathleen T, Sauer Tim D and Yorke James A, (1996) "Chaos : An Introduction to Dynamical System",p.359-362. 
7. Yue Wu, Xiaobing Zhou, Jia Chen, BeiHui, "Chaos synchronization of a new 3D chaotic system", Chaos, Solitons and Fractals 42 (2009) 1812-1819.

8. Sahab, A.R. and M. Haddad Zarif, Chaos Control in Nonlinear Systems Using the Generalized Backstopping Method, American J. of Engineering and Applied Sciences 1 (4): 378-383, 2008.

9. Wenjuan $\mathrm{Wu}$, Zengqiang Chen, Zhuzhi Yuan, The evolution of a novel four-dimensional autonomous system: Among 3-torus, limit cycle, 2-torus, chaos and hyperchaos, Chaos, Solitons and Fractals 39 (2009) 2340-2356.

10. Giuseppe Grassi , Damon A. Miller, "Synchronizing chaotic systems up to an arbitrary scaling matrix via a single signal", Applied Mathematics and Computation 218 (2012) 6118-6124

11. Ling-Dong Zhao a,b, Jian-Bing Hu b,n, Jian-An Fang a, Wen-Xia Cui a, Yu-Long Xu a, Xin Wang a, “Adaptive synchronization and parameter identification of chaotic system with unknown parameters and mixed delays based on a special matrix structure" ,ISA Transactions 52 (2013) 738-743.

12. Ali Reza Sahab and Mohammad Haddad Zarif, Improve Backstepping Method to GBM, World Applied Sciences Journal 6 (10): 1399-1403, 2009.

13. Chun-Lai Li, , Jian-Bin Xiong, Wen Li, "A new hyperchaotic system and its generalized synchronization",Optik 125 (2014) 575- 579.

14. Jianbing Hu, Lingdong Zhao, ZhenguangXie, "Control and synchronizing nonlinear systems with delay based on a special matrix structure", Commun Nonlinear SciNumerSimulat 19 (2014) 1072-1078.

15. Yongguang Yu, Han-Xiong Li, JianDuan, "Chaos synchronization of a unified chaotic system via partial linearization", Chaos, Solitons and Fractals 41 (2009) 457-463.

16. Hu JB, Han Y, Zhao LD. Synchronizing chaotic systems using control based on a special structure and extending to fractional chaotic systems Commun Nonlinear SciNumerSimul 2010;15:115-23. 